For comparison, Fig. 2 contains the evaluation of data measured on the $40 \%$ sample (dashed lines). Here again we get a straight line (Ornstein-Zernike behaviour) at $17^{\circ} \mathrm{K}$, but at $1.5^{\circ} \mathrm{K}$ the curve bends off, indicating that at this concentration (maximum of susceptibility at $13{ }^{\circ} \mathrm{K}$ ) there is a transition to another (longer-range) type of order.

Superconductivity does not seem to have any influence on the magnetic order in our sample. At the superconductive transition temperature of $2 \cdot 6^{\circ} \mathrm{K}$ neither the shape of the correlation function nor the correlation length changes. The short-range order found in our sample simply ignores superconductivity.

The absolute intensity has not been measured in our experiments, but we can get a rough estimate of the neutron intensity observed and thus of the magnetization fluctuation per $\mathrm{Tb}$ atom. In doing so we measure the neutron intensity for larger angles up to the first nuclear Bragg peak. (It should sit on a broad magnetic peak, which was not observed within our experimental accuracy.) Taking the Lorenz factor into account, we can use the nuclear peak (where the structure factor and the nuclear scattering lengths are known) to calibrate the integrated small-angle intensity. Doing so we find that the average $z$-compound per $\mathrm{Tb}$ atom is some tenth of a Bohr magneton (instead of 9 B.M. of the free ion). We cannot distinguish, however, whether the magnetic moment of the Tb-ion is depressed or whether the moments are less correlated.

\section{Discussion}

The superconductive coherence length of $\mathrm{Ce}_{0.8} \mathrm{~Tb}_{0.2} \mathrm{Ru}_{2}$ is not exactly known, but is certainly larger than the magnetic correlation length of $15 \AA$. Therefore it is still possible that the magnetic order in our sample is established by some kind of a Rudermann-Kittel interaction via the conduction electrons. In systems where the magnetic correlation length is larger than the coherence length it is expected that the correlation length changes when going through the superconducting transition. Looking for such systems should be a challenge for further investigations.

\section{References}

Hillenbrand, B. \& Wilhelm, M. (1970). Phys. Lett. 31 A, 448-449.

Matthias. B. T., Suhl, M. \& Corenzwit, E. (1958). Phy's. Ret. Lett. 1, 449-450.

Rupp, G. (1973). J. Phy's. C. In the press.

Schmatz, W., Springer, T., Schilten, J. \& Ibel, K. (1974). J. Appl. Cryst. 7, 96-116.

Steiner, P., Gumprecht, D. \& Hüfner. S. (1973). Phis. Rer. Le'tt. 30, 1132-1135.

Taylor, R. D., Dicklert, W. K., Erickson, D. J., Giorgi, A. L., Matthias, B. T. Olsen, C. E. \& Szklatz, E. G. (1972). Low Temperature Conference No. 13, Boulder, Colorado.

Willhelm, M. \& Hill.enbrand, B. (1970). Z. Naturforsch. 26a, 141-149.

\title{
Magnetic Properties in Partially Ordered $\mathrm{Ni}_{3} \mathrm{Mn}$
}

\author{
By U. Denkhaus, J. Schelten and W. Schmatz \\ Institut für Festkörperforschung der Kernforschungsanlage Jülich, Germany. (BRD)
}

\begin{abstract}
Hahn and Keller concluded from the magnetization curves of partially ordered $\mathrm{Ni}_{3} \mathrm{Mn}$ that magnetism is caused only by ordered clusters which are ferromagnetic and thermally unstable. This model was questioned by Paoletti and Ricci in 1967 who showed by scattering with polarized neutron scattering that the disordcred matrix also becomes magnetic below $400{ }^{\circ} \mathrm{K}$. In order to elucidate the behaviour of the matrix and its correlation to the clusters, an $\mathrm{Ni}_{3} \mathrm{Mn}$ alloy was investigated by neutron small-angle scattering at temperatures between 78 and $500 \mathrm{~K}$ with different applied magnetic fields. The small-angle scattering, which is only of magnetic origin, confirmed that the magnetization really consists of two contributions. One type of magnetization is localized at small ordered clusters and has a Curie temperature $T_{c}=750^{\circ} \mathrm{K}$ which is about the same as the ordering temperature. The other type of magnetization is localized in the environment of the ferromagnetic cluster and has a $T_{c} \simeq 400 \mathrm{~K}$. In order to interpret the scattering pattern of the clusters and to understand its temperature dependence it has been assumed, based on independent evidence, that the clusters are elongated particles of uniform size. In this case, the major axis is found to be about $40 \AA$, four times larger than the minor axes. A high un axial anisotropy with the two easy directions parallel to the long axis was verified because the scattering of the magnetic cluster did not depend on the strength of the applied field nor on the angle betwcen the scattering vector and the magnetic field. In the absence of an applied magnetic field the magnetization of the environment of a cluster has been found to be parallel to the magnetization of the cluster. An applied field of up to $6 \mathrm{kG}$ gradually turns this magnetization into a homogeneous magnetization of the matrix with its direction parallel to the external field. In contrast to this behaviour of the matrix, the magnetic moment of a cluster jumps only into that easy direction which is closest to the direction of the external field.
\end{abstract}

